\title{
Estudio de bioequivalencia de montelukast en tabletas masticables de 5 mg
}

\author{
Ángela Piedad Medina, Francisco Javier Olaya, Mónica Patricia Navas, \\ Ángela María Tilano, Enrique Muñoz \\ Delivery Technologies, S.A.S., Envigado, Colombia
}

\begin{abstract}
Introducción. La importancia de los medicamentos genéricos radica en la posibilidad de la disminución de los costos en el sistema nacional de salud, sin sacrificar la calidad del servicio ni la eficacia y la seguridad de los tratamientos.

Es importante resaltar que los estudios de bioequivalencia pretenden demostrar que los perfiles farmacocinéticos del producto de prueba y del producto de referencia son similares e intercambiables. El montelukast sódico está indicado para la profilaxis y el tratamiento crónico del asma, en adultos y pacientes pediátricos de 12 meses de edad o más. En general, es bien tolerado y las reacciones adversas son un poco más frecuentes en los pacientes tratados con el fármaco que en los tratados con placebo.

Objetivos. Comparar la biodisponibilidad de Amisped®, montelukast en tabletas masticables de 5 mg fabricadas por Sanofi-Aventis con la de Singulair $\AA$, montelukast en tabletas masticables de $5 \mathrm{mg}$ elaboradas por Merck Sharp \& Dohme.

Materiales y métodos. Se comparó la magnitud y la velocidad de la absorción de montelukast en 18 voluntarios sanos, empleando un diseño cruzado completo al azar. El bioanálisis de las muestras se hizo por cromatografía líquida de alta resolución.

Resultados. Los resultados para el genérico y el innovador, respectivamente, fueron: $\mathrm{T}_{\max }$ (horas) $2,17 \pm 0,73$ y $2,28 \pm 0,88 ; C_{\max }(\mathrm{ng} / \mathrm{ml}) 607,42 \pm 122,92$ y $627,69 \pm 134,17 ; \mathrm{AUC}_{0-\mathrm{t}}\left(\mathrm{ng}{ }^{\star} \mathrm{h} / \mathrm{ml}\right) 3.316,39 \pm 861,57$ y $3.545,40 \pm 1.070,07 ; A^{\prime} C_{0-\infty}\left(\mathrm{ng}^{\star} \mathrm{h} / \mathrm{ml}\right) 3.450,92 \pm 904,89$ y $3.722,03 \pm 1120,60 ; \mathrm{K}_{\mathrm{e}}(1 / \mathrm{h}) 0,25 \pm 0,05$ y

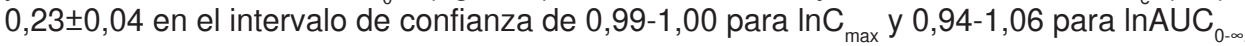

Conclusiones. La formulación ensayada de Amisped® de Sanofi-Aventis es bioequivalente a la formulación de referencia Singulair® de Merck Sharp \& Dohme.
\end{abstract}

Palabras clave: asma, medicamentos genéricos, medicamentos innovadores, equivalencia terapéutica.

doi: http://dx.doi.org/10.7705/biomedica.v32i3.708

\section{Bioequivalence study of montelukast $5 \mathrm{mg}$ chewable tablets}

Introduction. The importance of generic drugs is the possibility of reduced costs in the national health system without sacrificing quality of service and the efficacy and safety of treatments.

However, bioequivalence studies must show that the pharmacokinetic profiles of the test product and reference product are similar and interchangeable. Montelukast sodium is indicated for the prophylaxis and chronic treatment of asthma in adults and pediatric patients 12 months of age or older. It is generally well tolerated, although adverse reactions are more frequent in patients treated with the drug than in those treated with placebo.

Objectives. To compare the bioavailability of Amisped $^{\circledR}$ (5 mg montelukast chewable tablets) manufactured by Sanofi-Aventis and $5 \mathrm{mg}$ chewable tablet montelukast (Singulair ${ }^{\circledR}$ ) developed by Merck.

Materials and methods. The magnitude and rate of absorption of montelukast was compared in 18 healthy volunteers using a randomized complete crossover design. The bioassay was performed by high performance liquid chromatography.

Results. Results are indicated for the generic and innovator, respectively: $T_{\max }$ (h) $2.17 \pm 0.73,2.28 \pm 0.88$; $\mathrm{C}_{\max }(\mathrm{ng} / \mathrm{mL}) 607.4 \pm 122.9,627.7 \pm 134.2 ; \mathrm{AUC}_{0-\mathrm{t}}\left(\mathrm{ng}{ }^{*} \mathrm{~h} / \mathrm{ml}\right) 3,316 \pm 861,3,545 \pm 1,070 ; \mathrm{AUC}_{0-\infty}\left(\mathrm{ng}{ }^{*} \mathrm{~h} / \mathrm{ml}^{2}\right)$

\footnotetext{
Contribución de los autores:

Francisco Javier Olaya y Ángela Piedad Medina dirigieron el proyecto.

Mónica Patricia Navas dirigió la parte clínica.

Ángela María Tilano realizó los análisis estadísticos.

Enrique Muñoz asesoró el proyecto.
} 
$3,450 \pm 904,3,722 \pm 1121 ; \mathrm{K}_{\mathrm{e}}(1 / \mathrm{h}) 0.25 \pm 0.05,0.23 \pm 0.04$ in the confidence range of $0.99-1.00$ for $\mathrm{InC}_{\max }$ and 0.94-1.06 for InAUC ${ }_{0 . \infty}$.

Conclusions. The formula tested in Amisped ${ }^{\circledR}$ from Sanofi-Aventis is bioequivalent to the reference formulation of Merck Singulair ${ }^{\circledR}$.

Key words: Asthma; generic, drugs; reference drugs, therapeutic equivalency.

doi: http://dx.doi.org/10.7705/biomedica.v32i3.708

A los medicamentos innovadores o de marca se les realizan innumerables ensayos hasta demostrar su eficacia y seguridad en un largo proceso de investigación y desarrollo (preclínica, fases I, II y III), lo cual requiere una inversión grande y un tiempo de 7 a 15 años. Esta inversión la asumen las compañías multinacionales, las cuales hacen uso del derecho de patentar el principio activo y el proceso de fabricación, con el fin de recuperar la inversión y obtener beneficios.

Después del vencimiento de la protección proporcionada por la patente (20 años) al medicamento innovador, otras compañías farmacéuticas nacionales - multinacionales pueden tomar la invención y desarrollar otra formulación, otro proceso de fabricación, y solicitar permiso en el país respectivo para su comercialización. Siempre deben cumplir con los estándares nacionales e internacionales de calidad. Estos medicamentos son los denominados genéricos, los cuales deben ser, además, equivalentes farmacéuticos.

En el caso de los productos farmacéuticos sólidos administrados por vía oral, se necesita demostrar la equivalencia terapéutica para que sean intercambiables con el medicamento innovador. En general, a todos los medicamentos sólidos orales se les debe demostrar la equivalencia terapéutica, pero la diferencia radica en que algunos requieren solamente una demostración in vitro $\mathrm{y}$ otros la requieren in vivo, según su clasificación biofarmacéutica y con sus características farmacológicas, químico-físicas y farmacocinéticas.

Estos estudios son estudios clínicos de fase IV, específicamente, se llaman estudios de bioequivalencia. Un medicamento se convierte en "genérico" cuando al hacerse un estudio de biodisponibilidad comparativa frente al producto

Correspondencia:

Ángela Piedad Medina, Delivery Technologies, S.A.S., Carrera $48 N^{\circ} 48$ sur-75, interior 163, Centro Múltiple Las Vegas, Envigado, Colombia

Teléfono: (574) 4487935

angela.medina@deliverytechnologies.com.co

Recibido: 12/04/11; aceptado:20/04/12 innovador, se obtienen resultados similares en la evaluación de sus perfiles plasmáticos.

La importancia de los medicamentos genéricos radica en la posibilidad de la disminución de los costos en el sistema nacional de salud, sin sacrificar la calidad del servicio ni la eficacia y la seguridad de los tratamientos. Por lo tanto, es importante resaltar que los estudios de bioequivalencia pretenden demostrar, por medio de perfiles farmacocinéticos, que el producto de prueba o candidato a genérico y el producto de referencia o innovador son similares e intercambiables.

En Colombia, se han generado bastantes debates sobre los medicamentos genéricos, de los que se ha afirmado que presentan demasiados "problemas" e, incluso, algunos autores han llegado a la conclusión de que son inútiles y poco seguros (1, Isaza S. Ponencia de la Federación Médica Colombiana ante el foro "La verdad de los medicamentos genéricos" de las Comisiones Séptimas del H. Senado de la República y Cámara de Representantes. Bogotá, 21 de abril de 2009). Es en este punto donde cobran importancia los estudios de bioequivalencia, ya que con ellos se garantiza la eficacia y seguridad de los medicamentos a los cuales están accediendo miles de colombianos.

El montelukast sódico (figura 1) está indicado para la profilaxis y el tratamiento crónico del asma en adultos y pacientes pediátricos de 12 meses de edad o más. En general, es bien tolerado y las reacciones secundarias son un poco más frecuentes en los pacientes tratados con el fármaco que en los tratados con placebo (2).

El montelukast es un antagonista de los leucotrienos, similar al zafirlukast que presenta la ventaja de no inhibir las isoenzimas CYP2C9 y CYP3A4 del citocromo P450, por lo que presenta muchas menos interacciones que el zafirlukast con otros fármacos.

En muchos procesos alérgicos se liberan sustancias conocidas como leucotrienos, que ocasionan broncoespasmo, causando sibilancias y dificultad para respirar. El montelukast reduce la 


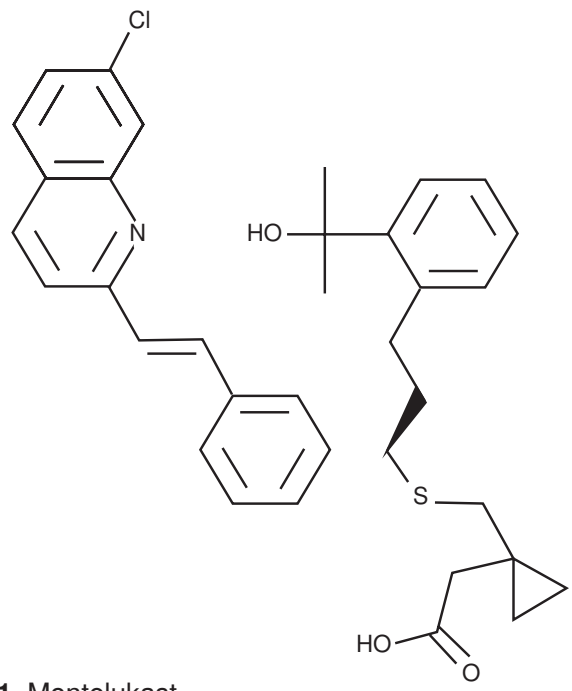

Figura 1. Montelukast

producción de leucotrienos, evitando el cierre de las vías respiratorias (2).

El montelukast se absorbe rápidamente tras la administración oral. Después de la administración de una tableta recubierta de $10 \mathrm{mg}$ a adultos en ayunas, la concentración plasmática máxima $\left(C_{\max }\right)$ se alcanza en tres a cuatro horas $\left(T_{\max }\right)$. La biodisponibilidad oral media es de $64 \%$. La biodisponibilidad oral y la $\mathrm{C}_{\max }$ no varían al ingerir por la mañana una comida estándar. Más de 99 \% del fármaco se une a las proteínas plasmáticas, el volumen de distribución en estado estacionario es de 8 a $11 \mathrm{~L}$ y es ampliamente eliminado. La farmacocinética del montelukast es casi lineal para dosis orales de hasta $50 \mathrm{mg}$ y es similar en hombres, mujeres, ancianos y jóvenes (3).

En este trabajo se comparó la biodisponibilidad en dosis únicas de Amisped®, montelukast en tabletas masticables de $5 \mathrm{mg}$, fabricadas por Sanofi-Aventis y comercializadas actualmente, contra el producto innovador también comercializado en Colombia, Singulair $\AA^{\circ}$, montelukast en tabletas masticables de $5 \mathrm{mg}$ fabricado por Merck Sharp \& Dohme (Registro sanitario INVIMA N ${ }^{\circ} 2008$ M-007543R1), empleando un diseño de tipo abierto, de asignación aleatoria, cruzado, en dos periodos, con siete días de lavado. La bioequivalencia se evaluó determinando los parámetros farmacocinéticos de los promedios de los perfiles sanguíneos de cada fármaco, el límite de confianza del $90 \%$ para los datos transformados (formulación de prueba/ formulación de referencia) $\left(\mathrm{C}_{\max }\right.$, area under the curve $(\mathrm{AUC})_{0 \rightarrow t}$, y $\mathrm{AUC0_{ \rightarrow \infty }}$ ), y la influencia de la secuencia, formulación y período, empleando análisis de varianza.

\section{Materiales y métodos}

\section{Selección de los voluntarios}

Se hizo una convocatoria en las diferentes universidades del área metropolitana, por medio del correo electrónico y la página web del laboratorio, de tal forma que las personas que estuvieron interesadas se inscribieron en una base de datos que contiene la información mínima para ser tenidos en cuenta de acuerdo con los criterios de inclusión.

Posteriormente, se hizo una reunión donde se les aclararon todos los temas generales relacionados con los estudios de bioequivalencia y, luego, los específicos relacionados con el fármaco montelukast, los objetivos del estudio y los riesgos potenciales de participar en él.

A los voluntarios preseleccionados (de acuerdo con las medidas antropométricas) se les realizó anamnesis, examen físico y los siguientes exámenes de laboratorio: hemograma completo, glucemia en ayunas, transaminasa glutámico oxaloacética, transaminasa glutámico pirúvica, colesterol total, creatinina sérica, triglicéridos séricos, urea en sangre, albúmina sérica, citoquímico de orina, prueba de VIH, prueba para antígeno de superficie de hepatitis $\mathrm{B}$ y prueba de embarazo.

\section{Criterios de inclusión y exclusión}

Los voluntarios fueron adultos sanos de ambos sexos con una edad comprendida entre 18 y 55 años, una relación peso/talla con una variación de $\pm 15 \%$ dentro del rango de normalidad, con historia médica sin antecedentes de enfermedad hepática, cardiaca, renal, sanguínea o del sistema nervioso central; signos vitales y presión sanguínea normales; ritmo cardiaco adecuado, y resultados de exámenes de laboratorio dentro del rango normal o dentro de una desviación considerada no significativa clínicamente por el director médico y el investigador principal; también, que no presentaran alergia al fármaco en investigación.

Se excluyeron los voluntarios que presentaron algún estado de enfermedad antes del estudio, historia de alcoholismo o adicción a drogas o que hubieran tomado algún medicamento (incluidas las vitaminas) dos semanas antes del estudio, los fumadores (más de 10 cigarrillos por día), con una dieta especial (por ejemplo, vegetarianos), y los que, antes del estudio hubieran donado más de $500 \mathrm{ml}$ de sangre en 14 días, $750 \mathrm{ml}$ en 30 días, $1.000 \mathrm{ml}$ en 90 días, $1.250 \mathrm{ml}$ en 120 días, 1.500 
$\mathrm{ml}$ en 180 días, $2.000 \mathrm{ml}$ en 270 días, y $2.500 \mathrm{ml}$ en un año.

El voluntario sería retirado del estudio en cualquiera de las siguientes circunstancias: un efecto adverso complicado, una violación al protocolo, retirada del consentimiento, terminación del estudio por el patrocinador o cualquier enfermedad sistémica ocurrida durante el periodo del estudio que requiriera otros fármacos.

\section{Aspectos éticos}

El estudio fue conducido de acuerdo con los principios de la Declaración de Helsinki (4) y la actual legislación colombiana en materia de ensayos clínicos (5)

El protocolo del estudio fue aprobado por el Comité de Bioética del Instituto de Investigaciones Médicas de la Facultad de Medicina de la Universidad de Antioquia (Acta No. 014. Aprobación del Protocolo del Estudio Montelukast, 5 de agosto de 2010).

Antes de iniciar su participación en el estudio, todos los voluntarios fueron informados, y firmaron un acta de compromiso y un consentimiento informado.

\section{Número de voluntarios}

El número de voluntarios se estableció con base en los coeficientes de variación para $C_{\max }$ y AUC, obtenidos en estudios previos con la misma molécula y aplicando la siguiente ecuación: $n_{1} \geq[t(a / 2,2 n-2)+t(\beta, 2 n-2)]^{2}\left[C V /\left(20-\theta_{0}\right]^{2}(6)\right.$.

El estudio se inició con 24 voluntarios; en el segundo ciclo, tres voluntarios pertenecientes a la secuencia R-T decidieron retirar su consentimiento para no continuar en el estudio. Por lo tanto, se retiraron tres voluntarios de la secuencia T-R aleatoriamente, para cumplir con un diseño balanceado; así, el análisis estadístico se llevó a cabo con 18 voluntarios sanos.

\section{Equivalencia farmacéutica}

La valoración (contenido) del principio activo en las formulaciones y el ensayo de disolución se determinaron siguiendo los métodos suministrados por Sanofi-Aventis. Para la evaluación de la uniformidad de dosis, se siguieron los lineamientos dados para este ensayo en la USP vigente.

\section{Diseño del estudio}

Se hizo un diseño cruzado completo al azar, de dosis única, con un periodo de lavado de siete días entre los dos periodos de dosificación. En cada uno de ellos, los voluntarios recibieron dos tabletas de la formulación de prueba o la formulación de referencia de montelukast en tabletas masticables de $5 \mathrm{mg}$.

Durante el estudio se estandarizaron la dieta y la ingestión de fluidos. Los voluntarios no consumieron alcohol, cualquier bebida o alimento que tuviera metilxantinas (cafeína, té, infusión de hierba mate, cola, Coca-Cola $\AA$, chocolate), desde las 48 horas previas a la administración del fármaco hasta las 24 horas posteriores a la última toma de muestra del segundo periodo. Además, no pudieron ingerir bebidas ni alimentos que tuvieran toronja o pomelo una semana antes de la administración del fármaco en el ciclo I hasta el final del estudio.

La ingestión de alimentos y bebidas fue idéntica en ambos periodos del estudio. Los sujetos permanecieron en ayunas de 10 a 12 horas antes de recibir el medicamento. El desayuno, el almuerzo y la cena fueron suministradas a las 4,6 y 12 horas, respectivamente, después de la ingestión del fármaco. Durante el estudio y las primeras 4 horas después de ingerido el medicamento, los voluntarios mantuvieron una postura sentada y sin inclinar el cuerpo exageradamente hacia delante para evitar comprimir los órganos de la digestión. Pasado este tiempo, tampoco se acostaron cabeza abajo (caída libre) o en posición fetal sobre el lado izquierdo que pudiera causar reflujo o disminuir la digestión.

Los medicamentos se administraron de acuerdo con el plan de asignación aleatoria por el personal autorizado, según los siguientes dos esquemas. Tratamiento $\mathrm{T}$ (ensayo): masticaron 15 veces (durante 30 segundos) dos tabletas de Amisped $\AA$, formulación de prueba de montelukast de $5 \mathrm{mg}$ por tableta, comercializado actualmente en Colombia, con administración de $240 \mathrm{ml}$ de agua a temperatura ambiente (cuadro 1). Tratamiento $R$ (referencia): masticadas 15 veces (durante 30 segundos) dos tabletas de Singulair $\AA$, formulación de referencia comercializada en Colombia, de Montelukast de 5 mg por tableta, con administración de $240 \mathrm{ml}$ de agua a temperatura ambiente.

\section{Recolección de las muestras sanguíneas}

Los voluntarios fueron internados 12 horas antes del comienzo del estudio, garantizando el control sobre el ayuno de 12 horas. A las 6:00 a.m. se les colocó un catéter intravenoso aséptico y se recolectó un total de 17 muestras de sangre a las 0 horas (antes de la administración de la formulación) 
y a las $0,5,1,1,5,2,2,5,3,3,5,4,4,5,5,6,8,10$, 12,14 y 24 horas, en tubos protegidos de la luz. Se centrifugaron y el plasma se almacenó en un congelador a $-20{ }^{\circ} \mathrm{C}$, hasta su posterior análisis (7-9).

\section{Método bioanalítico}

El bioanálisis de las muestras de montelukast se hizo por cromatografía líquida de alta resolución (HPLC) en un cromatógrafo líquido Shimadzu LC-2010 A HTTM con detector UV con longitud de onda variable. Las muestras se prepararon por extracción líquido-líquido.

Para el desarrollo del método bioanalítico, se tomaron como referencia los métodos reportados por Kitchen, et al., Alsarra, Sripalakit, et al., y otros (10-22).

En la validación del método bioanalítico, se determinaron los siguientes parámetros: selectividadespecificidad, precisión y exactitud (precisión intraensayo e interensayo), linealidad y rango, límite de detección, límite de cuantificación, porcentaje de recuperación, estabilidad del fármaco en plasma a $-20{ }^{\circ} \mathrm{C}$, temperatura ambiente y ciclos de congelación-descongelación.

Las muestras de plasma de cada voluntario, desde el tiempo cero $\left(T_{0}\right)$ hasta el último tiempo $16\left(T_{16}\right)$, se sometieron a análisis cromatográfico $y$, paralelamente con cada secuencia (corrida) de análisis, se corrieron una curva de calibración y muestras control a tres concentraciones diferentes (baja, media y alta).

Las curvas analizadas sirvieron como patrones de calibración del sistema cromatográfico y permitieron, además de aceptar o rechazar corridas o secuencias, cuantificar el principio activo en los voluntarios teniendo en cuenta el porcentaje de recuperación en plasma evaluado en la validación del método. Los resultados obtenidos en la validación durante el estudio cumplieron con los criterios de aceptación reportados (23).

\section{Análisis farmacocinético}

El análisis farmacocinético y la determinación de la bioequivalencia de montelukast consistió en el cálculo de los principales parámetros farmacocinéticos que definen la bioequivalencia en magnitud $\left(\mathrm{AUC}_{0-\infty}, \mathrm{AUC}_{0-\mathrm{t}}\right)$ y en velocidad $\left(\mathrm{C}_{\max }\right.$ y $\left.T_{\text {max }}\right)$.

Los parámetros farmacocinéticos se derivaron de las curvas de concentración en plasma Vs. tiempo. Los cálculos se hicieron con el paquete estadístico Minitab $^{\circledR}$, versión 14.

La concentración máxima $\left(\mathrm{C}_{\max }\right)$ y el tiempo máximo $\left(T_{\max }\right)$ se obtuvieron directamente de las curvas. El área bajo la curva desde tiempo cero hasta el último tiempo de muestreo $\left(A \cup C_{0-t}\right)$, se obtuvo mediante el método de los trapecios. El área bajo la curva desde tiempo cero hasta infinito $\left(\mathrm{AUC}_{0-\infty}\right)$, se calculó mediante la ecuación: $\mathrm{AUC}_{0-\infty}=$ $\mathrm{AUC}_{0-\mathrm{t}}+\mathrm{Ct} / \lambda z$, donde $\mathrm{Ct}$ es la última concentración cuantificable y $\lambda z$ es la constante de eliminación calculada por regresión lineal de la concentración $V s$. tiempo de la fase de eliminación. El tiempo de vida media $\left(\mathrm{t}_{1 / 2}\right)$ se calculó con la fórmula $\ln (2) / \lambda z$.

\section{Análisis estadístico}

Se hizo un test de ANOVA para In $\left(\mathrm{AUC}_{0-\infty}\right.$ y In $\left(C_{\max }\right)$, teniendo en cuenta las siguientes variables que podrían tener algún efecto sobre la respuesta principal: períodos, secuencia, sujetos y formulaciones.

Para concluir sobre la bioequivalencia entre ambas formulaciones, se empleó la prueba doblemente unilateral de Schuirmann (24-26) con los datos sin transformar y un intervalo de confianza de 90 $\%\left(\mathrm{IC}_{90 \%}\right.$ ) para los cocientes $\mu_{\mathrm{T}} / \mu_{\mathrm{R}}$ (formulación de prueba/formulación de referencia) $\left(\mathrm{C}_{\max }\right.$ y $\mathrm{AUC}_{0-\infty}$ de los datos transformados. Si el cociente así calculado y el intervalo de confianza se encontraban dentro del intervalo de bioequivalencia del 80 al $125 \%$, quedaba establecido que el producto era bioequivalente (27).

Cuadro 1. Identificación de las formulaciones bajo estudio

\begin{tabular}{lll}
\hline Identificación & Producto de prueba & Producto de referencia \\
\hline Nombre comercial & Amisped® & Singulair® \\
Forma farmacéutica & Tableta masticable & Tableta masticable \\
Cantidad de principio activo/tableta & 5 mg de montelukast & 5 mg de montelukast \\
Fabricante o distribuidor & Sanofi-Aventis & Merck Sharp \& Dohme \\
Número de lote & OCLO282 - 110809 & D013894 - E004971 \\
Fecha de vencimiento & $2012 / 04-2011 / 08$ & $2011-04$ \\
Sitio de fabricación & Cali, Colombia & Inglaterra \\
\hline
\end{tabular}


Se comprobaron los siguientes supuestos estadísticos: asignación aleatoria de las muestras, homogeneidad de las varianzas, linealidad del modelo estadístico, independencia y normalidad de los residuales, asignación aleatoria de los voluntarios.

\section{Resultados}

Este estudio de bioequivalencia fue conducido en 18 voluntarios colombianos, con resultados normales en el examen físico y el laboratorio clínico. El promedio de edad fue de $27,3 \pm 10,0$ años, el de la talla, de $1,7 \pm 0,1 \mathrm{~m}$ y el de peso, de $70,2 \pm 11,7 \mathrm{~kg}$.

Durante la ejecución del estudio un voluntario reportó en ambos ciclos un leve brote eritematoso en la frente y detrás de las orejas 12 horas después de ingerir el medicamento, el cual desapareció sin tratamiento alguno 12 horas después.

En general, no se observó ningún efecto adverso grave durante el desarrollo de este estudio.

Los resultados de los parámetros farmacocinéticos de $C_{\max }, T_{\max }, A \cup C_{0-t}, A \cup C_{0-\infty}, K_{e}$ yt $_{1 / 2}$, se presentan en el cuadro 2.

El perfil de concentración plasmática promedio (ng/ $\mathrm{ml}$ ) versus tiempo después de la administración de ambas formulaciones, se ilustra en la figura 2.

Los resultados del análisis de varianza (ANOVA) aplicado a In $\left(\mathrm{C}_{\max }\right)$ e In $\left(A \cup \mathrm{C}_{0-\infty}\right)$, para la relación análisis entre sujetos e intrasujeto (secuencia, ciclo, formulación, voluntarios) (cuadro 3), demostraron que estadísticamente no existían efectos debido a las secuencias y las formulaciones, lo que indicaba que las secuencias se comportaban homogéneamente. El análisis de varianza para

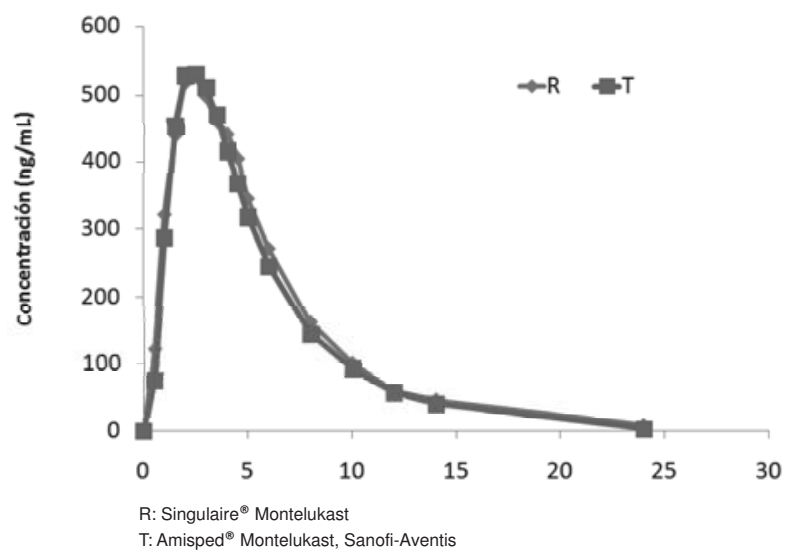

Figura 2. Perfil plasmático promedio de tabletas de $5 \mathrm{mg}$ de montelukast
Cuadro 2. Parámetros farmacocinéticos obtenidos para la formulación de ensayo $(T)$ y la formulación de referencia $(R)$

\begin{tabular}{lcc}
\hline Parámetros & \multicolumn{2}{c}{ Media \pm DE } \\
& Ensayo (T) & Referencia (R) \\
\hline $\mathrm{t}_{\max }$ (horas) & $2,17 \pm 0,73$ & $2,28 \pm 0,88$ \\
$\mathrm{C}_{\text {max }}(\mathrm{ng} / \mathrm{ml})$ & $607,42 \pm 122,92$ & $627,69 \pm 134,17$ \\
$\mathrm{AUC}_{0-\mathrm{t}}\left(\mathrm{ng}^{*} \mathrm{~h} / \mathrm{ml}\right)$ & $3316,39 \pm 861,57$ & $3545,40 \pm 1070,07$ \\
$\mathrm{AUC}_{0-\infty}\left(\mathrm{ng}^{*} \mathrm{~h} / \mathrm{ml}\right)$ & $3450,92 \pm 904,89$ & $3722,03 \pm 1120,60$ \\
$\mathrm{~K}_{\mathrm{e}}(1 /$ hora) & $0,25 \pm 0,05$ & $0,23 \pm 0,04$ \\
$\mathrm{t}^{\mathrm{t}}$ (horas) & $2,92 \pm 0,57$ & $3,08 \pm 0,63$ \\
\hline
\end{tabular}

Cuadro 3. ANOVA para $\ln \left(\mathrm{C}_{\max }\right)$ y $\ln \left(\mathrm{AUC}_{0-\infty}\right)$

\begin{tabular}{lcc}
\hline Fuente & \multicolumn{2}{c}{ Valor $\mathbf{p}$} \\
\hline Formulación & $\mathbf{I n}\left(\mathbf{A U C}_{\mathbf{0 - \infty}}\right)$ & $\ln \left({ }_{\mathrm{Cmax}}\right)$ \\
Ciclo & 0,162 & 0,896 \\
Secuencia & 0,839 & 0,039 \\
Voluntario & 0,099 & 0,146 \\
\hline
\end{tabular}

Ln: logaritmo neperiano

AUC: área bajo la curva

$\mathrm{C}_{\max }$ : concentración máxima

los ciclos o periodos, indicó que los efectos sobre la cinética de concentración obtenida en ambos ciclos era significativa para la $C_{\max }$, lo cual se puede presentar cuando existen diferencias en el manejo, análisis y almacenamiento de las muestras biológicas, diferencias climáticas, dietéticas o de actividad física y otras. En este estudio, el manejo y almacenamiento de las muestras fueron bien controlados, al igual que la dieta y la actividad física de los voluntarios. Sin embargo, el efecto periodo no tiene una incidencia significativa en el cálculo de la bioequivalencia, ya que la desviación estándar utilizada en el cálculo del intervalo de bioequivalencia no contiene la variabilidad dada por este efecto.

Los resultados obtenidos con la prueba doblemente unilateral de Schuirmann con los datos sin transformar y el intervalo de confianza de $90 \%$ para los cocientes $\mu_{\mathrm{T}} / \mu_{\mathrm{R}}$ (formulación de prueba/ formulación de referencia) $\left(\mathrm{C}_{\max }\right.$ y $\left.\mathrm{AUC}_{0-\infty}\right)$ de los datos transformados, se muestran en los cuadros 4 y 5 . El intervalo de confianza calculado para la relación de $\ln \left(\mathrm{C}_{\max }\right)$ y $\ln \left(\mathrm{AUC}_{0-\infty}\right)$, fue de 0,99-1,00 y 0,94-1,06, respectivamente.

\section{Discusión}

Antes del estudio in vivo, se pudo demostrar la equivalencia farmacéutica con la evaluación de la disolución, la uniformidad de dosis y la potencia 
Cuadro 4. Prueba doblemente unilateral de Schuirmann en 18 voluntarios sanos para la formulación test (ensayo) y referencia

\begin{tabular}{|c|c|c|c|c|c|}
\hline \multicolumn{6}{|c|}{ Prueba doblemente unilateral de Schuirmann } \\
\hline \multirow{2}{*}{$\begin{array}{l}\text { Parámetro } \\
\text { farmacocinético }\end{array}$} & \multirow{2}{*}{$\begin{array}{l}\text { Promedio producto } \\
\text { de prueba }(T)\end{array}$} & \multirow{2}{*}{$\begin{array}{c}\text { Promedio producto } \\
\text { de referencia }(\mathbf{R})\end{array}$} & \multirow{2}{*}{$\begin{array}{c}\text { Relación de } \\
\text { promedios (T-R) }\end{array}$} & \multicolumn{2}{|c|}{ Prueba de Schuirmann } \\
\hline & & & & $\mathrm{P}<80$ & $P>125$ \\
\hline $\mathrm{C}_{\max }$ & 607,42 & 627,69 & 20,27 & 0,008 & 0,008 \\
\hline$A \cup C_{0-\infty}$ & 3450,92 & 3722,03 & 271,11 & 0,01 & 0,01 \\
\hline Criterio & & & & $<0,05$ & $<0,05$ \\
\hline
\end{tabular}

$\mathrm{C}_{\max }$ : concentración máxima

$\mathrm{AUC}_{0-\infty}$ : área bajo la curva de cero a infinito

Cuadro 5. Intervalo de confianza del $90 \%$ en 18 voluntarios sanos para la formulación test (ensayo) y referencia

\begin{tabular}{|c|c|c|c|c|c|}
\hline \multicolumn{6}{|c|}{ Intervalo de confianza del $90 \%$} \\
\hline \multirow[t]{2}{*}{$\begin{array}{l}\text { Parámetro } \\
\text { farmacocinético }\end{array}$} & \multirow[t]{2}{*}{$\begin{array}{l}\text { Promedio producto } \\
\text { de prueba }(\mathrm{T})\end{array}$} & \multirow[t]{2}{*}{$\begin{array}{l}\text { Promedio producto } \\
\text { de referencia }(\mathbf{R})\end{array}$} & \multirow{2}{*}{$\begin{array}{c}\text { Relación de } \\
\text { promedios (T/R) } \\
(\%)\end{array}$} & \multicolumn{2}{|c|}{$\begin{array}{c}\text { Intervalo de } \\
\text { confianza }\end{array}$} \\
\hline & & & & Inferior & Superior \\
\hline $\ln \left(C_{\max }\right)$ & 6,39 & 6,42 & 99,53 & 99,8 & 100,2 \\
\hline $\ln (A \cup C)$ & 8,11 & 8,18 & 99,14 & 93,9 & 106,4 \\
\hline Criterio & & & & $>80$ & $<125$ \\
\hline
\end{tabular}

Ln: logaritmo neperiano

$\mathrm{C}_{\text {max }}$ : concentración máxima

$A \cup_{0-\infty}$ : área bajo la curva de cero a infinito

determinada por la valoración del producto de prueba, la cual no difirió en más de $5 \%$ con respecto a la del producto de referencia.

Aunque el montelukast en tabletas masticables es una forma farmacéutica diseñada especialmente para niños y este estudio de bioequivalencia se llevó a cabo con personas mayores de 18 años, los resultados obtenidos pueden extenderse a este grupo etario puesto que el estudio cumple con los requisitos metodológicos para demostrar bioequivalencia.

En el análisis estadístico de los datos no se observaron voluntarios con valores extremos en los parámetros farmacocinéticos de AUC y $\mathrm{C}_{\max }$. Asimismo, los datos encontrados concuerdan con los parámetros farmacocinéticos de magnitud y velocidad que se encuentran reportados $(3,9)$, de $535,73 \mathrm{ng} / \mathrm{ml}$ para $\mathrm{C}_{\max }$ en un $\mathrm{T}_{\text {max }}$ de $2,3 \pm 1,0$ horas, con un $\mathrm{t}_{1 / 2}$ entre 2,7 y 5,5 horas.

Se hicieron las transformaciones logarítmicas de las concentraciones de cada voluntario, la relación de los cocientes del medicamento genérico versus el innovador, y los intervalos de confianza $\left(\mathrm{IC}_{90 \%}\right)$, cumpliendo con los parámetros relevantes al determinar si dos productos son bioequivalentes. El intervalo de confianza calculado para la relación de In $\mathrm{C}_{\max }$ y In $\mathrm{AUC}_{0-\infty}$, se encuentran dentro del rango de aceptación establecido para poder concluir sobre la bioequivalencia en velocidad y magnitud de ambas formulaciones (FDA 0,8-1,25\%). Por lo tanto, se concluye que la formulación de Amisped®, montelukast en tabletas masticables de $5 \mathrm{mg}$, fabricada por Sanofi-Aventis, es bioequivalente a la formulación Singulair®, tabletas masticables de $5 \mathrm{mg}$ elaboradas por Merck Sharp \& Dohme.

Cada vez es mayor el interés en la comercialización y presencia en el mercado de los medicamentos genéricos y esto ha generado que la atención se centre en los procedimientos experimentales utilizados para la comparación entre el medicamento genérico y el de referencia.

La importancia de la incorporación de los medicamentos genéricos en el mercado farmacéutico radica en el costo notablemente inferior de un tratamiento con estos fármacos, sin querer significar esto que van a ser menos efectivos al innovador, pues su menor costo se debe a la menor inversión económica del laboratorio farmacéutico para su desarrollo y comercialización. Aquí es donde cobran importancia los estudios de bioequivalencia, ya que se hace necesario demostrar que la curva temporal de niveles plasmáticos del principio activo contenido en el medicamento genérico, es equivalente a la curva temporal obtenida con el medicamento de referencia. 
Los estudios de bioequivalencia se hacen obligatorios para medicamentos que presentan alto riesgo por sus características de toxicidad, margen terapéutico estrecho, clasificación biofarmacéutica, comportamiento farmacocinético y forma farmacéutica. Cabe anotar que, en Colombia, para el montelukastnoseexigenestudiosdebioequivalencia. Sin embargo, el laboratorio fabricante quiso llevar a cabo este estudio para poder demostrar su intercambiabilidad con el medicamento innovador.

Con los resultados obtenidos en este estudio de bioequivalencia, es posible inferir la equivalencia terapéutica del medicamento genérico (Amisped®) que se propone como intercambiable con el medicamento innovador (Singulair $\AA$ ). Dado que el principio activo en el medicamento genérico tiene el mismo perfil de concentraciones sanguíneas que en el medicamento innovador, se pueden considerar intercambiables, y la evidencia de eficacia clínica y seguridad del innovador se aplica al genérico.

\section{Conflicto de intereses}

En consideración a este trabajo, los autores convenimos de una forma conjunta establecer que no existen conflictos de intereses entre la empresa patrocinadora del mismo Sanofi-Aventis y Delivery Technologies, S.A.S., lo cual se certifica en el documento en el que el patrocinador autoriza la publicación.

También, los autores reconocemos y declaramos que no existe conflicto de intereses de tipo económico o de otro tipo, que pudiera suponer un sesgo para este trabajo.

\section{Financiación}

La empresa Sanofi-Aventis financió totalmente este estudio.

\section{Referencias}

1. Díaz G. La realidad de los medicamentos genéricos. Fecha de consulta: 20 de marzo de 2012. Disponible en: http:// gonzalodiaz.net/100/ley100/medesencial.shtml.

2. Hardman J, Limbird L. Goodman and Gilman. Las bases farmacológicas de la terapéutica. Novena edición. México, D.F.: Editorial McGraw-Hill; 1996.

3. Moffat AC, Osselton MD, Widdop B, Watts J. Clark's analysis of drugs and poison. London: Pharmaceutical Press; 2005. p. 1-2.

4. World Medical Association Declaration of Helsinki. Ethical principles for medical research involving human subjects. 59th WMA General Assembly, Seoul, October 2008. Fecha de consulta: 9 de marzo de 2010. Disponible en: http://www. wma.net/en/30publications/10policies/b3/17c.pdf.
5. Ministerio de Salud. Resolución No. 008430 DE 1993. Por la cual se establecen las normas académicas, técnicas y administrativas para la investigación en salud. Bogotá D.C.: Ministerio de Salud; 1993.

6. Liu JP, Chow SC. Sample size determination for the two one-sided tests procedure in bioequivalence. J Pharmacokinet Biopharm. 1992;20:101-4. http://dx.doi.org/10. 1007/ BF01143188

7. Chauhan B, Rani S, Nivsarkar M, Padh H. New liquid liquid extraction method for determination for montelukast in small volume human plasma samples using HPLC with fluorescence detector. Indian J Pharm Sci. 2006;68:517-20. http://dx.doi.org/10.4103/0250-474X.27834

8. Merck Sharp and Dohme de México. Diccionario de especialidades farmacéuticas. Fecha de consulta: 13 de mayo de 2010. Disponible en: http://www.facmed.unam.mx/ bmnd/plm_2k8/src/prods/36195.htm.

9. Patel NK, Subbaiah G, Shah H, Kundlik M, Sanyal M, Shrivastav PS. Rapid determination of Montelukast in human plasma by LC-ESI-MS/MS and its application to a bioequivalence study. Anal Lett. 2009;42:2041-59. http:// dx.doi.org/10.1080/00032710903082721

10. Kitchen CJ, Wang AQ, Musson DG, Yang AY, Fisher AL. A semi-automated 96 -well protein precipitation method for the determination of Montelukast in human plasma using high performance liquid chromatography/fluorescence detection. J Pharm Biomed Anal. 2003;31:647-54. http:// dx.doi.org/10.1016/S0731-7085(02)00723-9

11. Sripalakit $\mathbf{P}$, Kongthong B, Saraphanchotiwitthaya A. A simple bioanalytical assay for determination of Montelukast in human plasma: Application to a pharmacokinetic study. $J$ Chromatogr B Analyt Technol Biomed Life Sci. 2008;869:3844. http://dx.doi.org/10.1016/j.jchromb.2008.05.017

12. Liu L, Cheng H, Zhao JI, Rogers ID. Determination of Montelukast (MK-0476) and its S-enantiomer in human plasma by stereoselective high-performance liquid chromatography with column-switching. J Pharm Biomed Anal. 1997;15:631-8. http://dx.doi.org/10.1016/S0731-7085 (96)01894-8

13. Ochiai H, Uchiyama N, Takano T, Hara K, Kamei T. Determination of Montelukast sodium in human plasma by column-switching high-performance liquid chromatography with fluorescence detection. J Chromatogr B Biomed Sci Appl. 1998;713:409-14. http://dx.doi.org/10.1016/S03784347(98)00179-0

14. Al-Rawithi S, Al-Gazlan S, Al-Ahmadi W, Alshowaier IA, Yusuf A, Raines DA. Expedient liquid chromatographic method with fluorescence detection for Montelukast sodium in micro-samples of plasma. J Chromatogr B Biomed Sci Appl. 2001;754:527-31. http://dx.doi.org/10.1016/S03784347(01)00025-1

15. Smith GA, Rawls CM, Kunka RL. An automated method for the determination of Montelukast in human plasma using dual-column HPLC analysis and peak height summation of the parent compound and its photodegradation product. Pharm Res. 2004;21:1539-44. http://dx.doi.org/10.1023/ B:PHAM.0000041445.76931.27

16. Cheng H, Leff JA, Amin R, Gertz BJ, De Smet M, Noonan $\mathbf{N}$, et al. Pharmacokinetics, bioavailability, and safety of montelukast sodium (MK-0476) in healthy males 
and females. Pharm Res. 1996;13:445-8. http://dx.doi. org/10.1023/A:1016056912698

17. Ramakrishnan R, Migoya E, Knorr B. A population pharmacokinetic model for montelukast disposition in adults and children. Pharm Res. 2005;22:532-40. http://dx.doi. org/10.1007/s11095-005-2493-y

18. Al Omari MM, Zoubi RM, Hasan El, Khader TZ, Badwan AA. Effect of light and heat on the stability of Montelukast in solution and in its solid state. J Pharm Biomed Anal. 2007;45:465-71. http://dx.doi.org/10.1016/j. jpba.2007.07.014

19. Alsarra I, Al-Omar M, Gadkariem EA, Belal F. Voltammetric determination of montelukast sodium in dosage forms and human plasma. Farmaco. 2005;60:563-7. http://dx.doi. org/10.1016/j.farmac.2005.04.004

20. Radhakrishna T, Narasaraju A, Ramakrishna M, Satyanarayana A. Simultaneous determination of montelukast and loratadine by HPLC and derivative spectrophotometric methods. J Pharm Biomed Anal 2003;31:359-68.http://dx.doi.org/10.1016/S0731-7085 (02)00650-7

21. Alsarra IA. Development of a stability-indicating HPLC method for the determination of Montelukast in tablets and human plasma and its applications to pharmacokinetic and stability studies. Saudi Pharmaceutical Journal. 2004;12:136-43.
22. Papp R, Luk P, Mullett WM, Kwong E. A rapid and sensitive method for the quantitation of montelukast in sheep plasma using liquid chromatography/tandem mass spectrometry. J Chromatogr B Analyt Technol Biomed Life Sci. 2007;858:282-6. http://dx.doi.org/10.1016/j. jchromb.2007.08.003

23. Pérez A, Pujol F, Martí J. Validación de métodos analíticos. Barcelona: Asociación Española de Farmacéuticos de la Industria; 2001. p. 45-118.

24. Schuirmann DJ. A comparison of the two one-sided tests procedure and the power approach for assessing the equivalence of average bioavailability. J Pharmacokinet Biopharm. 1987;15:657-80. http://dx.doi.org/10.1007/ BF01068419

25. Hauschke D, Steinijansb V, Pigeot I. Bioequivalence studies in drug development methods and applications. Chichester: Wiley; 2007.

26. Zapater P, Horga JF. Bioequivalencia y genéricos. Los estudios de bioequivalencia I. Una aproximación a sus bases teóricas, diseño y realización. Rev Neurol. 1999;29:123546.

27. Food and Drug Administration. Guidance for Industry. Bioavailability and bioequivalence studies for orally administered drug. Silver Spring: Food and Drug Administration; 2006. p. 1-26. 